\title{
The Diagnosis of ADHD and in Children with Language Delay in Saudi Arabia: The Impacts and Consequences
}

Adil Alshammary*

School of Psychology and Clinical Language Sciences, University of Reading, UK

\begin{abstract}
The diagnosis of communication and behavioral difficulties in Saudi Arabia is currently still an emerging field and there are few governmental schools in the field of speech therapy in Saudi Arabia. These schools offer a bachelor degree in speech hearing rehabilitation, which started only a few years ago. For this reason, the number of Saudis certified as speech and language therapists is very limited, which has led to several issues regarding diagnosis, therapy and speech services in the kingdom being raised. First hand reports from trained speech and language practitioners suggest that it is common for children with a range of communication difficulties to receive a diagnosis of Autism Spectrum Disorder, with other internationally recognized diagnostic labels (e.g. Attention Deficit Hyperactivity Disorder; ADHD) being far less commonly used.

Due to limitation of number of certified professional speech language therapists in Saudi Arabia, a number of teachers, psychologists and even some religious individuals claim to be speech therapists. They provide unauthorized speech therapy sessions without any experience or permission, in non-authorized centres such as private schools, private centers and even in their homes. This study is aimed at highlighting the importance of accurate diagnosis and the impact of unauthorized workers on children with ADHD and autism inside Saudi Arabia.
\end{abstract}

Keywords: Autism; Attention deficit hyperactivity disorder; Speech therapy; Communication disorders

\section{Introduction}

Given the limited of number of certified professional speech and language therapists in Saudi Arabia, speech therapy services are currently struggling to provide comprehensive provision as well as to combat misdiagnosis and unauthorised care providers [1]. Moreover, a number of teachers, psychologists and even some religious individuals claim to be speech therapists. They provide unauthorised speech therapy sessions without any experience, in non-authorised centres, such as private schools, private centres and even in their homes. In other cases, individuals have established private schools for children with speech difficulties, Attention Deficit Hyperactivity Disorder (ADHD) or autism. However, many use their schools as business centres, recruiting non-professional teachers, often using celebrities and famous actors as an advertising tool for the school. Moreover, they may sell expensive products from unknown sources, such as nutrition for children with ADHD, autism or speech difficulties. Omega 3, low-calorie cookies and lactose free milk are just a few examples. Other individuals move to small rural cities to establish their own businesses and provide their own therapy, thereby being well away from governmental inspection.

As a result, children with speech and language problems can be misdiagnosed by these non-authorised individuals, with catastrophic consequences. With the advance of social media, such as Facebook and Twitter, many unauthorised speech therapists have acquired quite a level of fame. This scenario is also similar in other Gulf countries, which may partially explain the high reported prevalence of some disorders. Globally, the prevalence of children with ADHD rates between 3\% and $7 \%$. However, this figure in the Middle East is completely different from any other country in the world, with the prevalence being higher than for any other region. For instance, the prevalence of ADHD in United Arab Emirates is estimated to be 29.7\% [2] whilst other Middle Eastern countries do not yet have any reliable data about the occurrence of the condition. In a study by on ADHD in Saudi Arabia, it was estimated that the prevalence was approximately $16.4 \%$; however, this estimation was based on only one city (Dammam). In sum, the high prevalence of
ADHD in the Middle East should be considered with caution, due to the healthcare context described in the introduction.

Reports about autism in the Middle East and especially in Saudi Arabia are very rare. However, three research studies about autism in the Gulf countries are available and its occurrence in Arab countries. In one case, the estimated prevalence rate of autism in the United Arab Emirates (UAE) is approximately 58 per 10,000 children (with more autistic boys than girls). However, another study in Oman showed much lower autism prevalence among Omani children, with the estimated prevalence rate being 1.4 cases per 10,000 children. In Saudi Arabia, the prevalence of autism is unclear, especially for females, due to social pressures that lead to autistic females being easily hidden from external researchers [3]. Moreover, female children have more ability to hide their behavioural disorders compared to males. Also, the cultural environment may play an important role, for instance, some Saudi families in some regions pay more attention to their male children rather than females in terms of their development. Finally, there is a lack of willingness in some Saudi regions to report certain behaviours exhibited by females due to the cultural perspective of that region [4] (Figures 1-3).

Some studies have indicated that the symptoms of ADHD are similar to those of autism. Regarding which, they have shown that the two conditions have several common cognitive and behavioural symptoms, such as in relation to social and motor skills. These

${ }^{*}$ Corresponding author: Adil Alshammary, School of Psychology and Clinical Language Sciences, University of Reading, UK, Tel: +44 (0)118 378 8523; E-mail: a.m.a.alshammary@pgr.reading.ac.uk

Received April 02, 2018; Accepted April 10, 2018; Published April 18, 2018

Citation: Alshammary A (2018) The Diagnosis of ADHD and in Children with Language Delay in Saudi Arabia: The Impacts and Consequences. J Ment Disord Treat 4: 160. doi:10.4172/2471-271X.1000160

Copyright: @ 2018 Alshammary A. This is an open-access article distributed under the terms of the Creative Commons Attribution License, which permits unrestricted use, distribution, and reproduction in any medium, provided the original author and source are credited. 


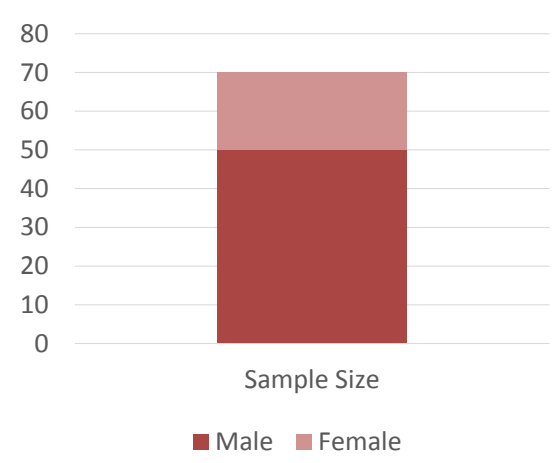

Figure 1: Size of the sample and gender of participants in the study.

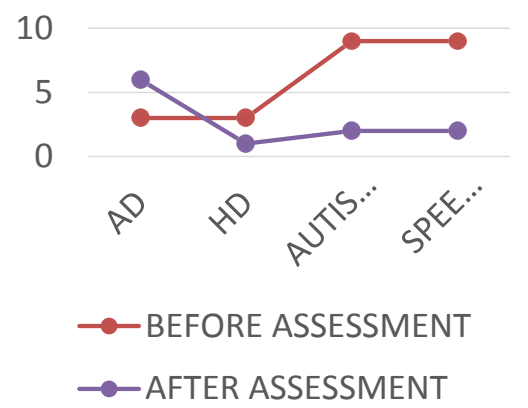

Figure 2: Changes in the quantity of disorders pre and post assessment (with VAS \& $A B C$ ) among the study sample.

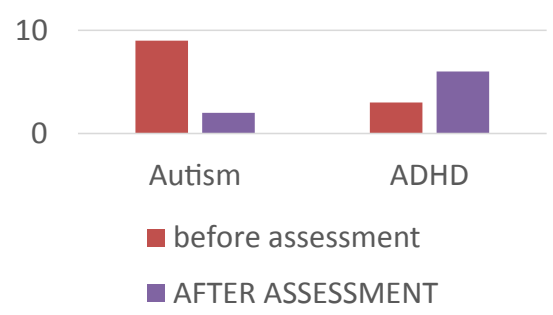

Figure 3: Comparison between Autism and ADHD before/ after assessment (with VAS \& ABC) among the study sample.

symptoms may occur due to genetic influence in both disorders [5]. That is, researchers have identified particular genes that could be common genetic etiological factors for ADHD and autism. According to this genetic study, ADHD and autism have a link in a specific region on chromosome 16p13. Another study was applied to 674 twins and also reported shared genetic influences between ADHD and autism [6].

This study is aimed to profiling the attention behaviour and communication abilities of a group of children, who have been given a diagnosis of autism Also, the study tries to highlight the importance of accurate diagnosis and the impact of unauthorized workers on children with ADHD and autism inside Saudi Arabia.

\section{Procedure and Methodology}

The study's primary methodology was the administration of a parent questionnaire, to be filled in by parents of children who had been given a diagnosis of autism. Question items were taken from two checklists (Vanderbilt Assessment Scales for ADHD and Autism
Behavior Checklist) available in the English language, which were combined and then translated into Arabic. Because this study was purely exploratory, the items from the questionnaire were not used for diagnostic purposes, but rather, clusters and patterns of behaviours were analysed from items across the two checklists. The combined questionnaires were completed with a pen and paper. For parents with low literacy ability, the questionnaire items were read aloud to them in a quiet location that was not overheard.

The choice of parents for the intervention was restricted to those registered at the Speech and Language Clinic in King Saud Medical City (KSMC), who had children with a communication disorder and questionable autistic behaviours. The age of these children ranged between five and nine years old (median $=6$ years old) and the sample size was 70 (50 boys). All the families had reports for their children stating that the cause of the communication disorder was autism and all the children had also had several speech sessions in the speech clinic. In order to have clear view about the study, an information sheet was provided in Arabic and English for the participants explaining the nature of their participation, prior to signing their informed consent. The consent forms were also produced in the two aforementioned languages. The researcher had previously worked in the same clinic and he contacted the potential participants by email, inviting them join the study. The email contained an explanation of the purpose of the project with a copy of the participant information sheet.

\section{Results}

The study findings show that two-thirds of the study participants demonstrated high reported levels of inattention behaviours in their daily activities. In terms of hyperactive behaviours, a third of the participants manifested high reported levels, while approximately a quarter of the participants showed moderate levels of hyperactive behaviours in their daily activities.

Also, the findings reveal that the majority of the participants manifested low levels of conduct problems, with approximately just $11 \%$ of participants scoring highly in this domain. This level of incidence is in line with the findings of Hamed [2], who reported that $13.4 \%$ of the 305 children in their sample showed signs of behavioural disorders. In terms of anxiety behaviours, no single case in the group study was reported to demonstrate those listed in the questionnaire. This finding diverges from that of Hamed [2], as well as in relation to Amr [3] study of 60 children from Al-Ahsa (Saudi Arabia), Amman (Jordan) and Al-Mansoura (Egypt), which both reported that children with ADHD and/or ASD had a higher risk of psychosocial problems, such as depression and anxiety.

In terms of school and academic performance, all participants in this study were reported as exhibiting problematic levels of performance. $20 \%$ of the participants who were reported as showing high levels of autistic behaviours also scored highly for inattention behaviours. This finding may indicate some phenotypic overlap between attentional and autistic behaviours. It is in partial agreement with a study by Sinzig, et al. [5] who found that in a sample of 83 children with ASDs and ADHD, there was a high phenotypic overlap between the groups, with two subtypes emerging: inattentive-stereotyped and the hyperactivecommunication impaired. The results demonstrate overlapping between inattention and autistic behaviors in children with speech difficulties in Saudi Arabia. At the level of the individual participants, previous medical reports had stated that the participants of this study fitted a diagnosis of autism and whilst $60 \%$ reported high levels of inattention, only low levels of autistic had been observed. 
Citation: Alshammary A (2018) The Diagnosis of ADHD and in Children with Language Delay in Saudi Arabia: The Impacts and Consequences. J Ment Disord Treat 4: 160. doi:10.4172/2471-271X.1000160

Page 3 of 3

\section{Discussion}

The results of the study demonstrate an overlap between inattention behaviour and autistic behaviour in children with speech difficulties in Saudi Arabia. At the level of individual participants, despite previous medical reports stating that the participants fitted a diagnosis of autism, $60 \%$ of participants in this study reported high levels of inattention, yet low levels of autistic behaviours. There were also $20 \%$ of participants that weren't reported to show high levels of inattention, autistic, hyperactivity, conduct problems, oppositional defiance or anxiety behaviour. Those $20 \%$ of participants only appeared to manifest problems in academic and classroom performance. These findings highlight the need for further attention to accurate differential diagnosis in the Saudi Arabian context and more research should be conducted into the reasons why children are struggling at school. Moreover, there is clearly a need for general criteria for speech and language clinics in Saudi Arabia, as well as increased awareness among parents about checking the credentials of the practitioners they are intending to see, and more publicly accessible information about key signs/symptoms of different diagnostic labels.

\section{Funding Acknowledgements}

Financial support was provided by King Saud Medical City, Riyadh, Saudi Arabia

\section{References}

1. Al Jadid MS (2011) Rehabilitation medicine in the Kingdom of Saudi Arabia Saudi Med J 32: 962-963.

2. Al Hamed JH, Taha AZ, Sabra AA, Bella H (2008) Attention Deficit Hyperactivity Disorder (ADHD) among Male Primary School Children in Dammam, Saud Arabia: Prevalence and Associated Factors. J Egypt Public Health Assoc 83 165-182.

3. Amr M, Raddad D, El-Mehesh F, Mahmoud EH, El-Gilany AH (2011) Sex differences in Arab children with Autism spectrum disorders. Res Autism Spectr Disord 5: 1343-1350.

4. Matson JL, Rieske RD, Williams LW (2013) The relationship between autism spectrum disorders and attention-deficit/hyperactivity disorder: an overview. Res Dev Disabi 34: 2475-2484

5. Sinzig J, Walter D, Doepfner M (2009) Attention deficit/hyperactivity disorder in children and adolescents with autism spectrum disorder: symptom or syndrome? J Atten Disord 13: 117-126.

6. Salhia HO, Al-nasser LA, Taher LS, Al-khathaami AM (2014) Systemic review of the of autism in Arab Gulf countries, Neurosciences Riyadh 19: 291-296. 